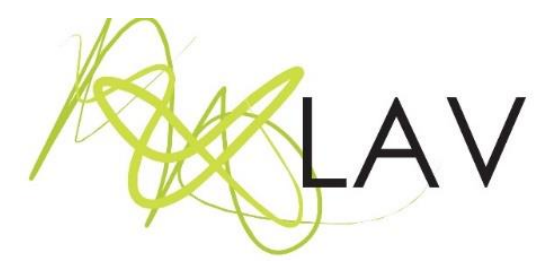

\title{
Encuentros que afectan y generan saber pedagógico entre docentes a través de cartografías visuales
}

Encounters that affect and generate pedagogical knowledge among teachers through visual cartographies

Fernando Hernández-Hernández

Grupo de Investigación Esbrina

Universidad de Barcelona

\section{Resumen}

Este artículo es parte de una investigación sobre cómo aprenden los docentes de secundaria. Una peculiaridad de este proyecto es que cuestiona diferentes dualidades características de la investigación cualitativa: la relación entre investigadores e investigadores se asume desde la trama de relaciones y no desde una distancia separadora; la generación de evidencias se lleva a cabo a partir de una experiencia de configurar cartografías visuales que permiten expandir sentidos y cuestionar el dualismo entre imagen y palabra; el análisis no pretender extraer una verdad de los datos por parte del investigador, sino se configura como un proceso de pensar que permite, a partir de conceptos, investigar desde la teoría; la propia noción de 'datos' es revisada, pues no se considera algo que 'está ahí fuera' sino que se genera en los encuentros; finalmente, la investigación no termina sólo en publicaciones académicas, sino en nuevos encuentros con docentes, donde la práctica de la formación se realiza para generar saber pedagógico de manera compartida. De una de estas experiencias de formación se da cuenta en este texto. Experiencia que ponemos en relación con la noción de 'afectos' que se sitúa en las intra-acciones relacionales que generan transformaciones en los docentes.

Palabras-clave: investigación post-cualitativa, afectos, investigar desde la teoría, cartografías, saber pedagógico, aprender docente.

\begin{abstract}
This article is part of a research project on how secondary school teachers learn. A peculiarity of this project is that it questions different dualities typical of qualitative research: the relationship between researchers and researched is assumed from a plot of relationships and not from a separating distance; the generation of evidence is carried through an experience of configuring visual cartographies that allow to expand meanings and question the dualism between images and words; the analysis does not pretend to extract a truth from the data, but it is configured as a process of thinking on concepts that allows to research from theory; the very notion of 'data' is revised, as something is not considered as 'out there' but it is generated in the encounters among actors; finally, the research does not end only in academic publications, but in new meetings with teachers, where the practice of teachers development is carried out to generate pedagogical knowledge in a shared way. One of these training experiences is the focus of this paper. This experience is related to the notion of 'affection' which is situated in a network of relational intra-actions that generate transformations in the participant actors.
\end{abstract}


Keywords: post-qualitative research, affections, research from theory, cartographies, pedagogical knowledge, teacher's learning.

Enviado en: 01/06/18 - Aprobado en: 17/07/18

\section{Esbozar el territorio}

En el grupo de investigación del que formo parte, Esbrina, tratamos de comprender cómo los sujetos, en su diversidad, transitan por las 'ecologías del aprender" (COLL, 2013; ERSTAD; SEFTON-GREEN, 2013; ERSTAD et al., 2016). En este trayecto hemos explorado: las experiencias de aprender de quienes no siguen las expectativas de la Escuela (HERNÁNDEZ, 2011); qué tipo de sujeto se educa en la escuela primaria (HERNÁNDEZ, 2010); cómo los jóvenes transitan en su aprender dentro y fuera de los centros (HERNÁNDEZ, 2017) y, actualmente, cómo los docentes transitan en sus procesos de aprender (HERNÁNDEZ-HERNÁNDEZ; SANCHO-GIL, DOMINGOCOSCOLLOLA, 2018). En este recorrido hemos comprendido que aprender y aprendizaje, como señala Atkinson (2011), no es lo mismo. Aprender tiene que ver con lo que nos afecta y, por ello, nos lleva a cambiar nuestra mirada sobre nosotros, los otros y el mundo. En este marco, como señala este autor, el aprender real se configura como parte de un evento que nos transforma. El segundo, refleja una fantasía de la pedagogía y la psicología, desde la que se proyecta la promesa de 'medir' lo que se aprende a través de pruebas de papel y lápiz. Esta visión, que es hoy hegemónica, tiene su reflejo en la industria escolar y el interés por la clasificación de alumnos, escuelas y países.

Para adentrarnos en los sentidos del aprender de los docentes, planteamos un proyecto de investigación APREN-DO: "Cómo aprenden los docentes: implicaciones educativas y retos para afrontar el cambio social" (Ministerio de Economía y Competitividad/EDU201570912-C2-1-R) donde profesores de secundaria fueron invitados a generar cartografías visuales de esos escenarios dentro y fuera de la Escuela donde aprenden, y a pensar en lo que valoran como fuente de conocimiento y experiencia. Les pedimos que crearan una cartografía visual sobre sus trayectorias de aprender y que participaran en conversaciones para generar formas de comprensión de sus desplazamientos de aprendizaje nómadas (BRAIDOTTI, 2006, 2014), sus tensiones y expectativas. En esta investigación, utilizamos las cartografías visuales como estrategia de pensamiento para explorar las transiciones de los profesores y las experiencias de aprender nómadas. La 
cartografía visual se entiende como una epistemología narrativa creativa y artística y una metodología de investigación que permite explorar intersticios, desplazamientos, viajes inestables, formas de conocimiento, ensamblajes y enredos a través de los cuales los profesores realizan sus trayectorias de aprendizaje (PAULSTON; LIEBMAN, 1994; RUITENBERG, 2007; ULMER; KORO-LJUNGBERG, 2015).

Desde el principio adoptamos "una forma artística de las ciencias sociales, una forma de representación radicalmente interpretativa" (RICHARDSON; ST. PIERRE, 2005, p. 964) inspirada en enfoques post-cualitativos (ST. PIERRE, 2011; JACKSON; MAZZEI, 2012; LATHER, 2013; LATHER; ST. PIERRE, 2013), que conforman un movimiento no unificado que cuestiona la noción misma de investigación: ya no se trata de obtener resultados sino de generar y poner en acción conceptos como rizoma, intensidad, afecto, gesto, desplazamiento, etc., que nos ayudan a pensar en cómo el aprendizaje se realiza a través de los movimientos y trayectorias de los docentes. Mediante la creación de cartografías visuales, como epistemología artística, indagamos en los intersticios, desplazamientos, viajes inestables, formas de conocer, ensamblajes y enredos a través de los cuales los profesores exploran y llevan a cabo sus itinerarios de aprender.

La investigación se llevó a cabo en tres centros de secundaria del área de Barcelona con 29 profesores voluntarios de secundaria. Un grupo de investigadores interactuó y ayudó a los docentes a construir sus cartografías. Todo el proceso fue grabado en vídeo y se tomaron notas de campo. Al terminar sus cartografías, cada docente fue grabado en vídeo explicando el proceso de realización de su cartografía. Pero este grupo no será el foco de este artículo. Lo será un grupo de docentes, asesores y administradores, involucrados desde hace varios años en una experiencia de generación de saber pedagógico (ABAKERLI BAPTISTA, 2014), y conocedoras de esta investigación por parte de quien escribe este artículo. Quienes integran este grupo expresaron su deseo de formar parte de una experiencia de creación y puesta en común de cartografías visuales de sus trayectorias biográficas de aprender. En este proceso compartieron no sólo los contenidos y movimientos colocados en las cartografías y en el proceso de toma de decisiones, sino también los aspectos transformativos de esa experiencia de aprender en compañía. El sentido las cartografías como desencadenantes de movimientos, intensidades y afectos es lo que presentamos a continuación.

\section{Cartografías y especialidad de la experiencia}

Cada cierto tiempo, en el campo de las ciencias sociales y de la educación, llega un nuevo giro a la agenda de la investigación. Este giro puede ser ocasional o más 
permanente. Este parece ser el caso del giro espacial, que desde la Geografía implica una reelaboración de la noción y significado de la 'espacialidad' (WARF; ARIAS, 2008, p. 1, parafraseado). Desde este marco, llegamos en nuestra investigación a las cartografías visuales, tanto como referente epistemológico como estrategia de investigación rizomática, con una trayectoria reconocida en las ciencias sociales y en la investigación educativa (PAULSTON; LIEBMAN, 1994; RUITENBERG, 2007; MCKINNON, 2011; ULMER; KORO-LJUNGBERG, 2015). Las cartografías visuales tienen también una presencia consolidada en los mapas - míticos, relacionales, espaciales, topológicos- (Biblioteca Nacional de España, 2017) y en las prácticas artísticas (CAIXAFORUM, 2012). En uno y otro caso representan territorios físicos, mentales y emocionales, así como exploran cuestiones sociales y políticas, experiencias corporales y vitales, y mapean lo intangible, los lugares vacíos (de no saber) y generan conceptos.

Estas nociones y prácticas de la cartografía las hemos puesto en relación con el enfoque de Guattari de las "cartografías esquizoanalíticas" (GUATTARI, 2000), consideradas como mapas que rechazan un dominio fijo e invariable de la subjetividad. Cartografías que son configuraciones relacionales, que cambian de estado y de estatus en función de ensamblajes particulares. Como Deleuze, Guattari considera los mapas como espacios de devenir, donde el inconsciente es "descubierto" a través de performances cartográficas (ULMER; KORO-LJUNGBEG, 2015, p. 139). Guattari contribuye de esta manera a los debates en curso en la investigación social y educativa, con respecto a la abstracción, el afecto, la cartografía, la subjetividad y la teoría.

A partir de esta revisión llegamos a una noción de cartografía como "aparato de captura" que territorializa lo nuevo y lo singular y puede mostrar ensamblajes, "multiplicidades o agregados de intensidades" (DELEUZE; GUATTARI, 1980, 2004, p. 15). Este enfoque nos ha llevado a considerar la cartografía como una evocación poderosa y versátil de trayectorias de aprender personal/social; como un conector de experiencias y conocimientos de diseño, abstracción y traducción que localizan los movimientos de la experiencia de aprender, así como un incrementador de conocimiento y apreciación hacia uno mismo y hacia el propio entorno en el que el aprender tienen lugar (ONSÉS, 2014). En ese sentido, las cartografías no son sólo una estrategia visual que posibilita relatos y experiencias, sino un espacio de enredo en el que todas estas sustancias - cuerpos y cosas, textos y situaciones, afectos e intensidades, movimientos y encrucijadas, ideas y 
formas de hacer, etc.- permanecen reunidas, dentro de una ontología procesual, relacional y performativa del devenir (ATKINSON, 2012). Uno de los conceptos que permite pensar estos movimientos es el de afecto.

\section{Cartografías, docentes y afectos: transitar de la investigación a la vida de los docentes}

Lo escrito hasta ahora es, en parte, la ruta por la que hemos transitado, los caminos que hemos recorrido en la investigación con la que este artículo se relaciona, en la que nos hemos planteado, siguiendo la propuesta de que investigar, como hacer filosofía, es generar conceptos y no buscar resultados (DELEUZE; GUATTARI, 1991, 1993). O como diría Teresa Kaufman (2011) generar modos de pensar y no resultados en forma de conocimientos. Algo que tan querido esto último por la ola neoliberal que domina en el mundo académico. Pero para poder pensar hay que situar los movimientos de la experiencia desde la cual trato de pensar el lugar de los afectos en las relaciones y el aprender de los docentes.

En la experiencia de compartir relacionada con la experiencia de creación de cartografías visuales participaron 11 docentes (que trabajan en diferentes ámbitos del sistema educativo) y quien esto escribe. Se han realizado seis encuentros y queda uno pendiente de cierre, en el que se pretende recoger lo que nos ha afectado del encuentro con las cartografías y relatos de los otros. Además de para configurar una cartografía conjunta, hecha de retazos de los encuentros celebrados hasta el momento. Lo que supone transitar por un continuo de relaciones.

1. En la primera sesión, sentados en torno a una mesa, compartimos el significado que dábamos a la noción y la práctica cartográfica y a la idea de aprender como un recorrido de lugares, encuentros y desplazamientos. Tras una intensa conversación, planificamos el próximo encuentro, al que aportaríamos materiales (objetos, memorias visuales y textuales..., ) para configurar las cartografías. En esta primera reunión se comenzó a esbozar la idea de que realizar las cartografías en grupo, desde la cercanía de unos y otros, podía ser lo que Bakko y Merz (2015) denominan una 'práctica afectiva', en las que las relaciones, los cuerpos y la memoria de lo vivido se pondrían en juego.

2. En el segundo encuentro, que tuvo lugar en un aula de diseño de la Facultad de Bellas Artes, con meses y taburetes, donde cada participante eligió un lugar - sobre una mesa o en el suelo - donde desarrollar la cartografía. Mientras el proceso avanzaba, todos nos movíamos, compartiendo impresiones y reflexiones y abriéndonos al diálogo sobre lo que cada uno estaba haciendo. Así, lo que podría haber sido un proceso individual se 
convirtió - como es característico de la vida de este grupo - en un lugar de encuentro y cuidado. Todo el proceso fue grabado en vídeo, fotografiado y se tomaron notas de campo para construir una memoria colectiva de este evento formativo de aprendizaje (ATKINSON, 2012).

3. En la tercera y cuarta reuniones, cada persona participante presentó el significado y las decisiones tomadas sobre la cartografía que había hecho. El resto del grupo interactuó, compartiendo resonancias y 'wonderings' (GREENBLATT, 1992). Estas conversaciones polifónicas se grabaron en vídeo y se transcribieron los intercambios verbales. Estas transcripciones fueron incluidas en un registro, con testimonios fotográficos de la realización de las cartografías de cada persona del grupo y los detalles visuales fotográficos tomados durante estas presentaciones al grupo. Gestos de cercanía y cuidado reflejan cómo lo que se compartía 'afectaba' a cada cual. Estas sesiones tuvieron lugar en una amplia sala de la Facultad de Bellas Artes. Lo que permitió colocar todas las cartografías en las paredes, como si se tratara de una galería de arte, pudiendo estar de pie y conversar en torno a cada cartografía.

4. En la quinta sesión, las docentes continuaron compartiendo las resonancias y 'wonders' generadas en cada una de ellas durante el proceso de construcción de las cartografías, así como el valor de compartir con los demás. Previamente a la reunión recibieron un documento que contenía la transcripción e imágenes de todo el proceso.

5. En la sexta reunión, hablamos de los principales aspectos que se destacaron y de cómo les había afectado todo el recorrido realizado. En este encuentro se configuran significados 'corpóreos', relacionales y biográficos como constitutivos de sus trayectorias de aprendizaje. Después de esta sesión, y después de compartir sus primeras resonancias y 'wonders', nos planteamos las siguientes preguntas, como punto de partida para seguir nuestro proceso de indagación:

¿Cuáles son los aportes de las estrategias artísticas y visuales de las cartografías a la narración de las trayectorias del aprender biográfico de los docentes?

¿Cómo se ven afectados los docentes por el desplazamiento generado por este movimiento de Investigación Artística? 
Fernando Hernández-Hernández

¿De qué manera el proceso cartográfico afecta las miradas sobre el aprender, las relaciones y las posicionalidades de los docentes?

Estas cuestiones, que se concretan en esta fase del proceso, están presentes desde el inicio del recorrido y son las que me llevan a pensar sobre y desde los afectos.

\section{Pensar el aprender desde los afectos}

Señalaba más arriba que aprender tiene que ver con 'sentirse afectado'. Un sentirse afectado que constituye un movimiento vinculado a la capacidad de existir en un tránsito entre estados. A este tránsito Spinoza lo denominó como afectos, que Camps (2011, p. 65) considera como intrínseco a la naturaleza humana, tan inevitables como el respirar, el crecer y el morir. En esta idea de afectos, la aportación de Spinoza es la que abre el camino para explorar su sentido. Spinoza entiende afectos como las afecciones del cuerpo, por las cuales aumenta o disminuye, es favorecida o perjudicada, la potencia de obrar de ese mismo cuerpo, y entiendo, al mismo tiempo, las ideas de esas afecciones. Así pues, si podemos ser causa adecuada de alguna de esas afecciones, entonces entiendo por «afecto» una acción (SPINOZA, 1677, 1980). De origen y naturaleza de los afectos: Def. III, énfasis en original). Deleuze y Guattari retoman la perspectiva de Spinoza, especialmente cuando dice que a cada relación de movimiento y de reposo, de velocidad y de lentitud, que agrupa una infinidad de partes, corresponde un grado de potencia. A las relaciones que componen un individuo, que lo descomponen o lo modifican, corresponden intensidades que lo afectan, aumentan o disminuyen su potencia de acción, que proceden de las partes exteriores o de sus propias partes (DELEUZE; GUATTARI, 1980, 2004, p. 260-261). De esta manera los afectos "son las fuerzas, intensidades, o capacidades de moverse y ser movido" (CVETKOVICH, 2012, p.134). Esto es lo que le sucede a Carmina, cuando ante su cartografía (figura 1) señala lo que le sucede cuando se mueve hacia una relación de aprender que le permite descubrirse en el otro:

Cuando yo doy este paso para querer conocer al otro que tengo allí delante, me pongo en disposición de aprender más. Yo me doy cuenta de que cuando yo soy capaz de romper estas barreras que nos hacemos, descubrimos niños, descubrimos personas. Entonces, ellos también comienzan a interaccionar contigo ... y a partir de ahí se crean situaciones muy bonitas. Y cambios también en estos niños. que hay en la escuela tan difíciles. 


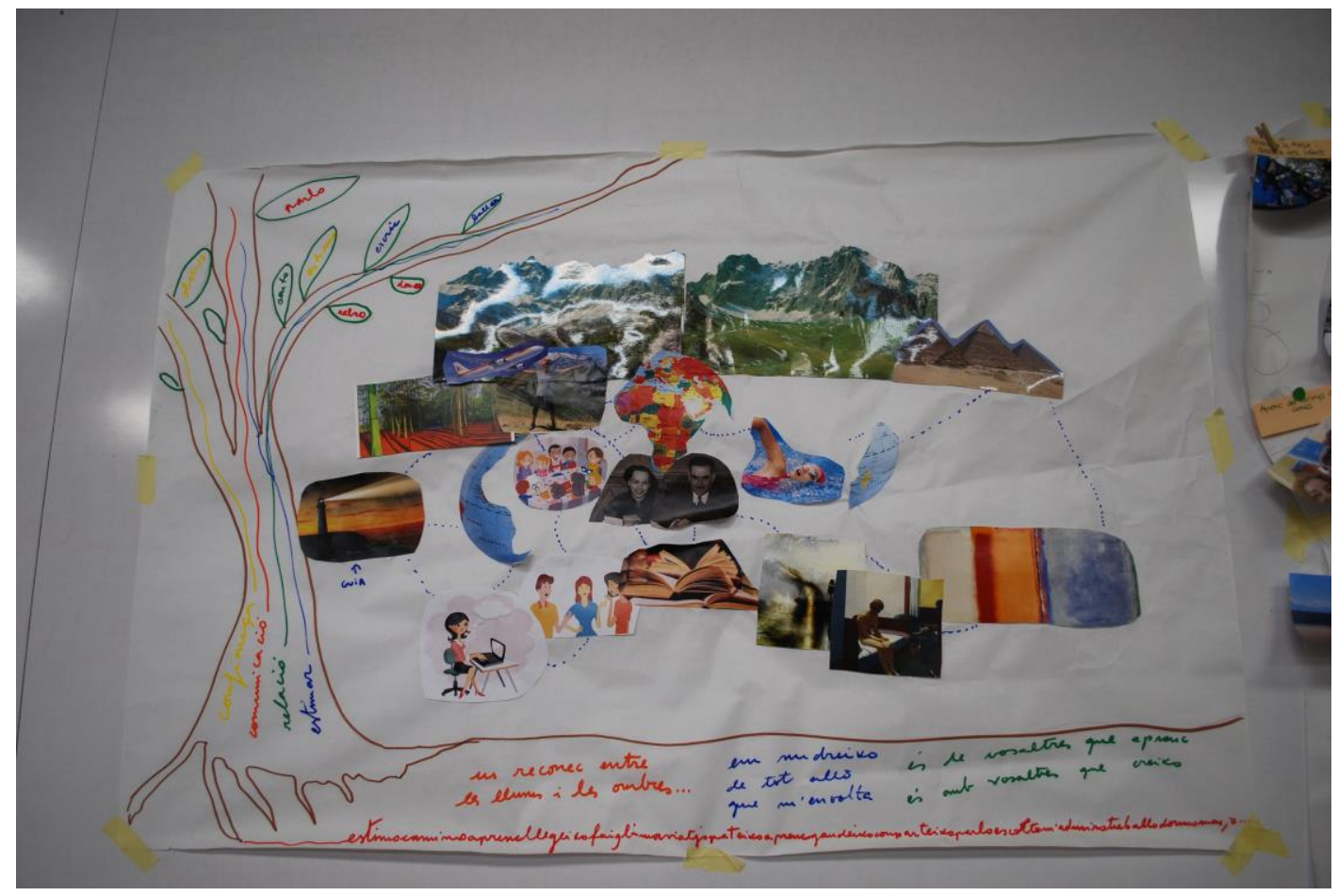

Figura 01. La cartografía de Carmina fotografiada por Nelly Alfandary.

$Y$ es que el afecto no es una fuerza que esté aislada, sino que es transversal ya que cruza todas las dimensiones del conocimiento, del sujeto como individuo y de la realidad. Además, implica un cambio sustancial en las relaciones en torno a la política, la investigación y la pedagogía. Pues afecto es "lo que separa al hombre dueño de sí del esclavo es la capacidad para transformar [...] efectivamente, solo un afecto más potente vence a otro afecto más inmediato y tentador" (CAMPS, 2011, p. 86-87).

Por eso, a la hora de pensar los afectos lo hacemos en el contexto de relaciones, prestando atención a las experiencias de estar implicados, a las posibilidades de hacer tangible la tensión que se proyecta en la capacidad crítica de reflexión (sobre lo que nos afecta). Es esta capacidad la señala Ita frente a la trama de relaciones de su cartografía (figura 2)

Esta idea de aprender me llega un momento en que yo aprendo en compañía. Los retos como motor de cambio han sido muy importantes en mi vida. Los retos me han incomodado y esta incomodidad me ha representado estar con vosotros, estar con otros grupos, estar en momentos que te han puesto y te han interpelado. A mí me han servido 
para romper ideas estereotipadas, para romper la utilización de imágenes estereotipadas y para romper la idea de prácticas estereotipadas con los niños.

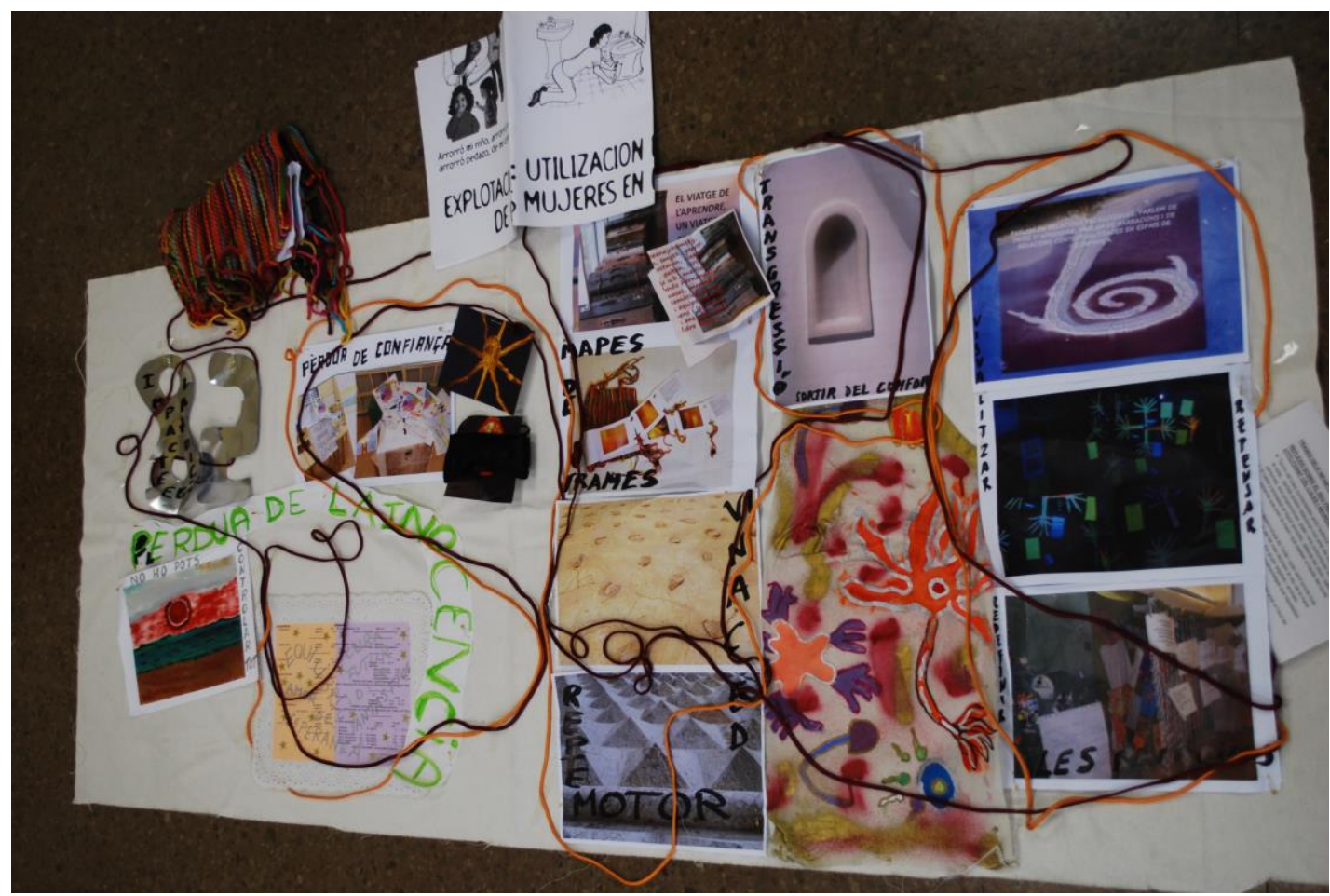

Figura 02. La cartografía de Ita fotografiada por Nelly Alfandary.

Desde esta aproximación, el afecto sería una fuerza natural invisible (a diferencia de las emociones que son visibles) que preceden a la individualidad, y que posiciona a la relacionalidad (siempre hay un otro) como eje ontológico. Los afectos, por tanto, producen y reconocen transformaciones, como parte de una intra-acción relacional. Lo que significa prestar atención a los movimientos de afecto que se generan en las relaciones. No como categorías o indicadores pre-definidos (como sucede con las emociones) sino como 'emergencias' que, a través de los recuerdos de lo vivido (de la experiencia), visibilizan lo que ha afectado. Esto permite, como señala Spinoza, transformar los afectos que impiden actuar en afectos que potencian la acción. Y es esta capacidad transformadora "lo que separa al hombre (y la mujer) dueño de sí del esclavo" (CAMPS, 2011, p. 84).

Los afectos se hacen visibles en las cartografías, en los encuentros con los docentes en nuestro trayecto de relaciones, no como algo fijo que se puede atrapar como si un indicador fuera, sino que se transforma en las relaciones de la investigación y los encuentros que propicia: con la cartografía, con los otros, con los textos. Es lo que hace 
Juanjo (figura 3) cuando, después de señalar una serie de movimientos biográficos que le han afectado en su proceso de aprender, apunta una relación con la información y el conocimiento que ahora proyecta en su modo de ser maestro y de afectar el aprender de los jóvenes:

Aquí representaba algunas de las ideas que me han acompañado. Todo el tema de relación con las noticias, con el mundo, con el conocimiento, que me han acompañado siempre. Una de las ideas que me han acompañado sería la de aprendiendo sobre el mundo, aprendiendo sobre las personas, aprendiendo sobre nosotros mismos al mismo tiempo. Y también aprender para comprender este mundo, estas personas $y$ comprendernos también a nosotros.

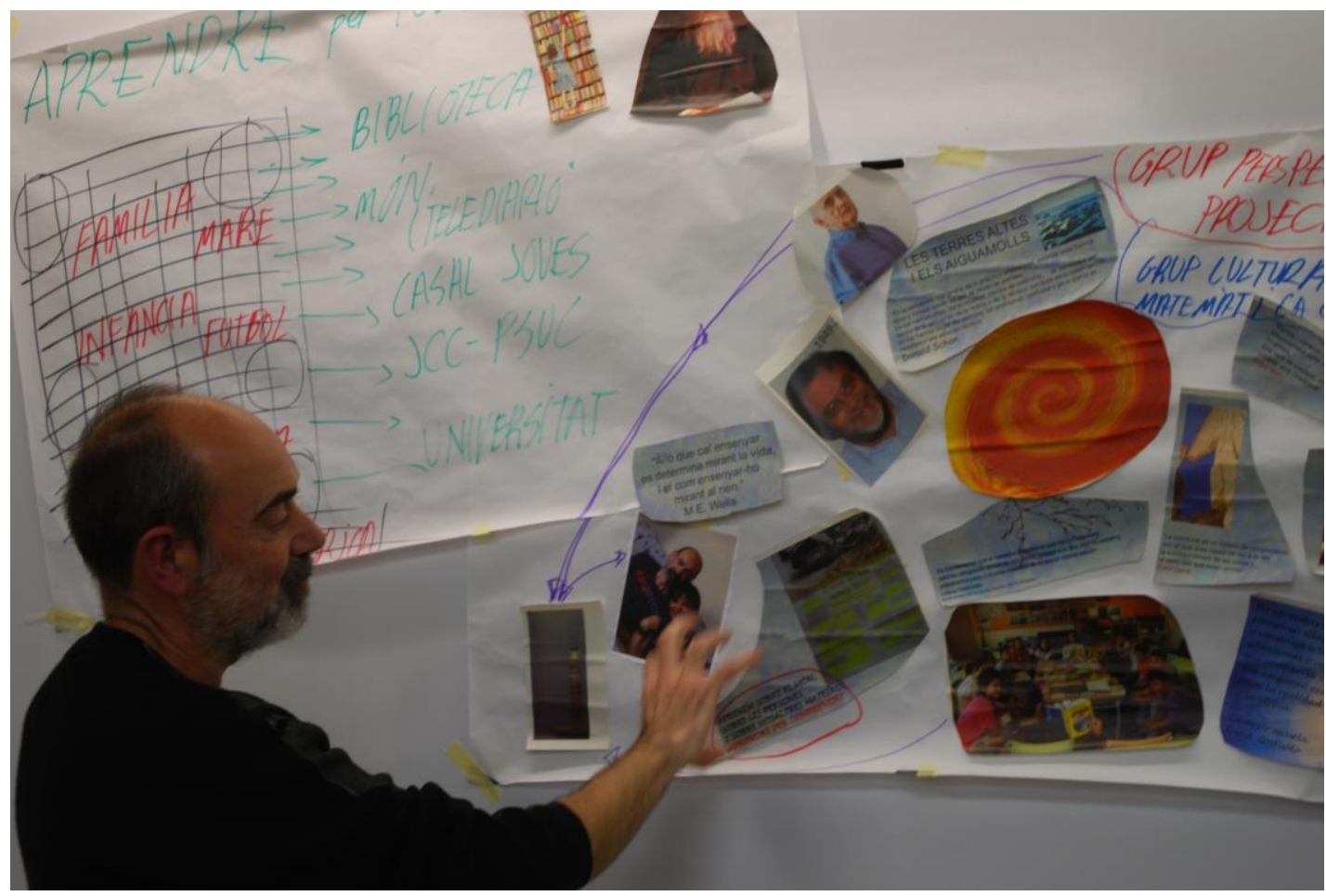

Figura 03. Juanjo presentando su cartografía. Fotografía realizada por Nelly Alfandary.

Los afectos son aquello que, al ser esenciales en el ser humano, permiten el movimiento en un cuerpo cambiante. Son aquella fuerza invisible in-corporada en un devenir corporal, en una expresión de un encuentro entre una forma corpórea y fuerzas que no 
son necesariamente "humanas" (HICKEY-MOODY, 2016, p. 260) que, situada en un momento concreto y en una situación relacional, desborda de tal manera que permite un desplazamiento de un estado a otro. El afecto es, por tanto, flujo y reflujo, como el ciclo de la marea, se transforma a sí mismo y transforma lo que le rodea y encuentra nuevos significados, aplicaciones y potencialidades a través de su uso [...] es una materialidad que siempre ha estado y está en proceso (BAKKO; MERZ, 2015, p. 8). Marisol muestra este desplazamiento en sus afectos de aprender, cuando lo asocia con una obra de Mieireles (figura 4) desde la que reverbera la conciencia de trama:

He querido situar que yo aprendo en forma de rizoma y por eso el rizoma de Deleuze me acompaña, y comienzo a caminar con esta idea de rizoma. "Pie detrás de pie, no hay otra manera de caminar". $Y$ entonces este rizoma está representado con una obra de Cildo Meireles que se llama "la malla de la libertad", y esta metáfora también me acompaña hace tiempo, como esta idea que pongo en el centro cuando digo "somos trama, yo aprendo haciendo trama y siendo sentido que soy trama" y que en esta trama estoy en tránsito. Y también quería ubicar esta idea de esta posición de movimiento, de aprender en movimiento, en tránsito, en posición nómada.

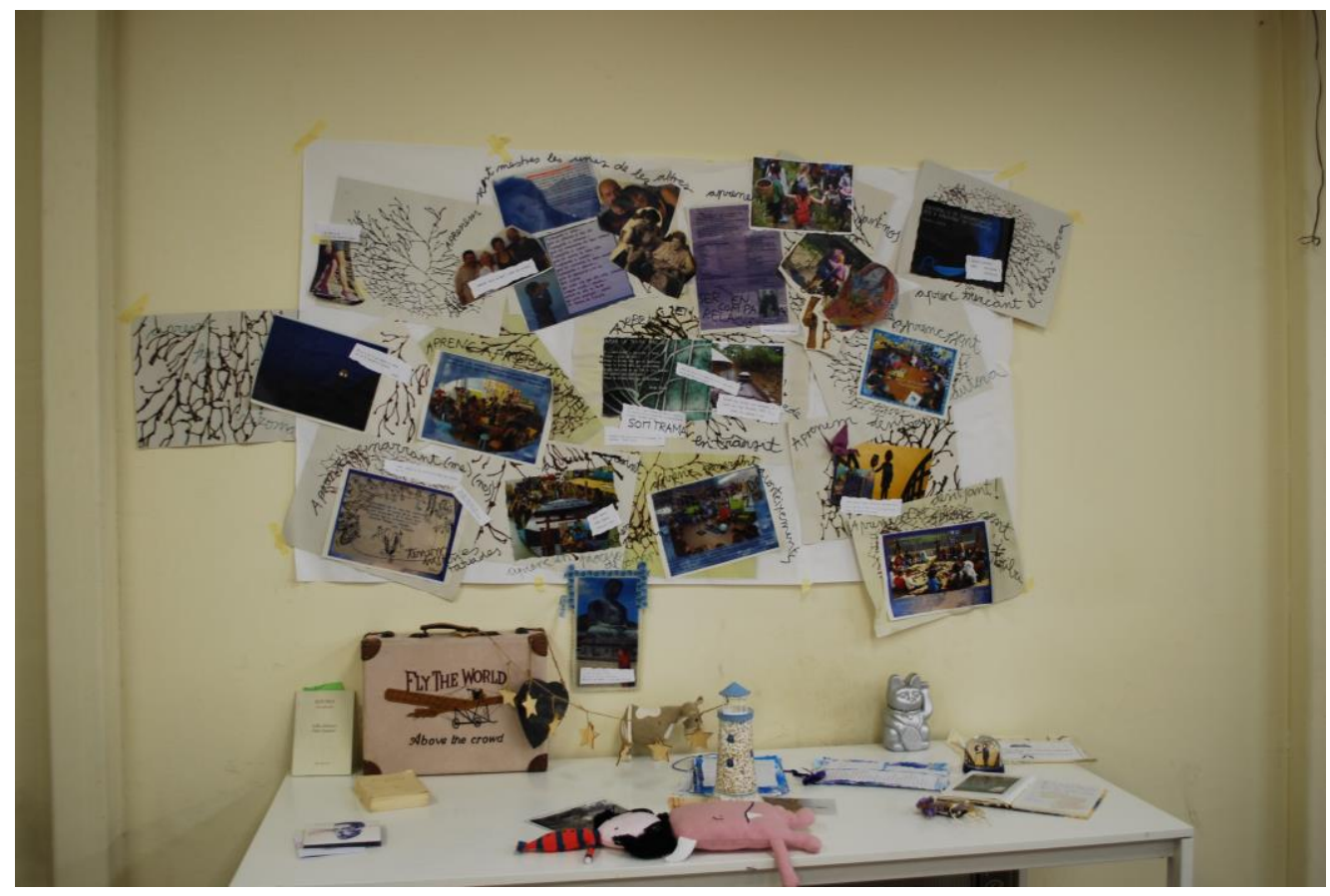

Figura 04. La cartografía de Marisol. Fotografia realizada por Nelly Alfandary.

Decía más arriba que una forma de localizar los movimientos y temporalidades de los afectos es hacerlo a través de 'las prácticas afectivas'. Una de las que aparece en el 
compartir de las personas del grupo es el dejarse afectar por las palabras de los otros. Sumergirse en la escucha para aprender a nombrar(se) de otra manera. Es lo que hace Mar (figura 5) cuando pensando en voz alta ante su cartografía relaciona la experiencia de aprender en compañía en el grupo de adultos con las que tuvo antes, cuando participaba en un grupo de tiempo libre con otros jóvenes:

Me di cuenta de que cojo también palabras vuestras, que estoy aprendiendo y pienso: son conversaciones, son movimientos, son momentos de compartir, donde se crean momentos únicos que nos hacen saltar, nos hacen como saltar de nivel e ir progresando. Las abejas (en su cartografía) supongo que tienen un movimiento, se relacionan, se comunican, informan, tienen toda una serie de códigos ... Pensé que yo en espacios así de iguales ... muy importante fue el espacio, de estar a un 'esplai', de hecho, fundamos uno. Yo estaba en el de jóvenes y fundamos el de pequeños y fue un lugar de crecimiento brutal, entre iguales, entre mis amigos y compañeros, $y$ entre los chicos que también estaban.

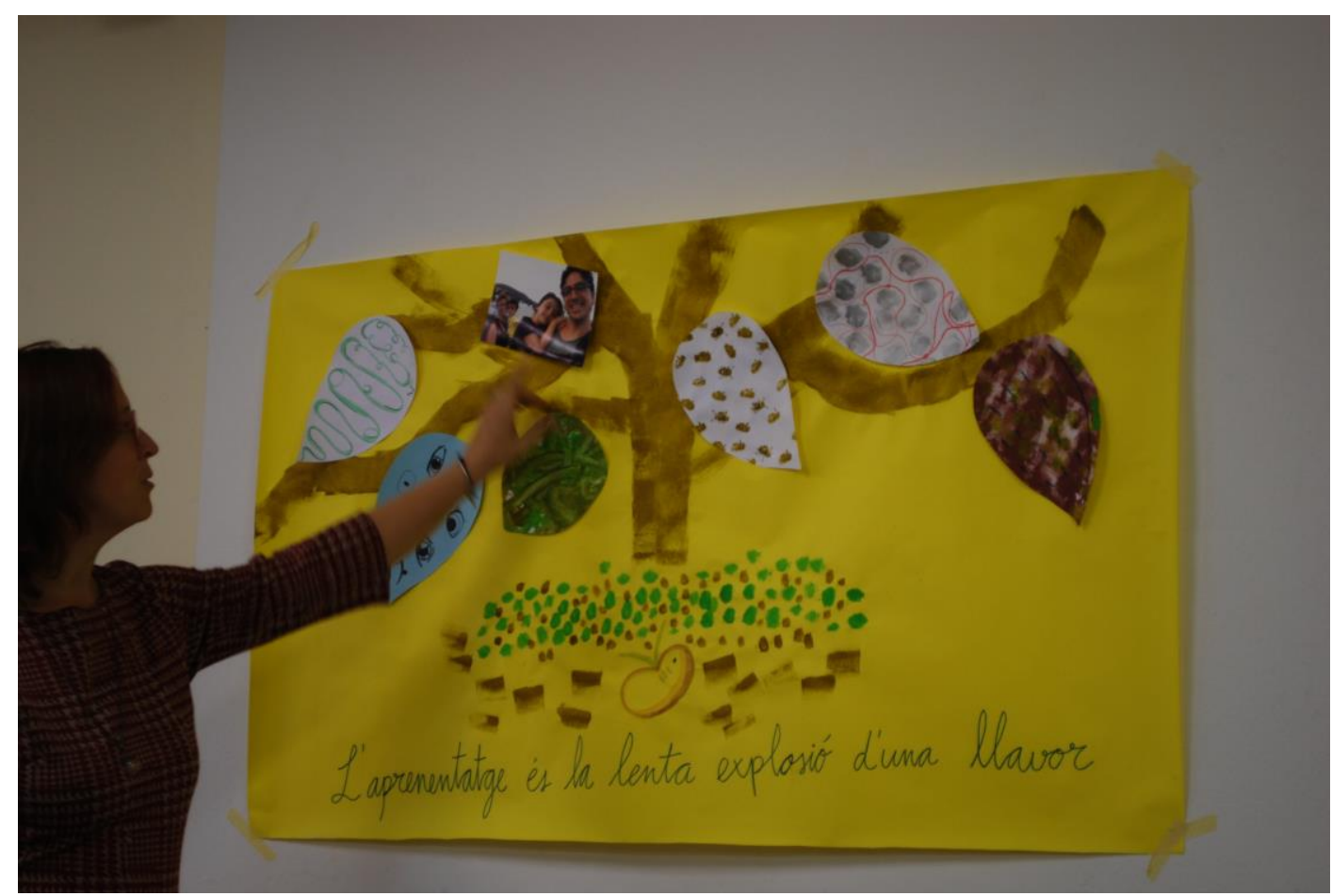

Figura 05. Mar presentando su cartografía. Fotografía realizada por Nelly Alfandary

De esta manera el afecto se nos aparece en las intra-acciones, donde la fuerza que hace que nos relacionamos es el afecto. Lo que posibilita, como vemos en este recorrido que si 
hablamos de afectos es porque nos permiten pensar las relaciones en torno al aprender desde otra ontología y epistemología. Por tanto, redefine un lenguaje, planteando un decir nuevo. Convirtiéndose, como señalan Bakko y Merz (2015, p. 8) "en una fuerza de indeterminación social que nos ofrece la oportunidad de mirar lo que es, imaginar lo que podría ser en su lugar, y comprender que este 'en su lugar' siempre está sucediendo".

\section{Una pedagogía de los afectos}

Al pensar desde el espacio de encuentro y de los afectos que propició la realización de las cartografías, pudimos localizar momentos, relaciones y experiencias de aprender, pero no cómo el aprender tiene lugar. Las cartografías de los docentes cuentan historias sobre dónde, con quién y con qué aprenden, pero no está claro -es desconocido (unknown) qué nos cuentan sobre cómo aprenden. Sin embargo, no consideramos este 'desconocido' (unknown) como una limitación, sino como una posibilidad de "ser constantemente desafiados por las dudas sobre lo que no sabemos". Esto es lo que hace la investigación afectiva, "que nos ayuda a ver que la incertidumbre y la curiosidad no sólo motivan nuevas investigaciones, sino que también inspiran impulsos artísticos" (SULLIVAN; GU, 2017, p. 50).

De este recorrido se deriva también lo que podría esbozarse como una pedagogía de los afectos, desde donde se promueve una crítica afirmativa por aproximación y no por negación, desde los afectos que se movilizan en las relaciones. Siendo el foco de la atención las relaciones (las intra-acciones) y no lo que le pasa a cada individuo.

En este caso, las cartografías visuales no son sólo una forma de expresión visual y textual, sino un proceso de colaboración y generación de conceptos que van más allá de la representación visual. Para los docentes e investigadores, esto significa, "en cierto modo, ponernos a nosotros mismos en peligro, no ser reconocidos dentro de los marcos normalizadores que rigen nuestra práctica" (ATKINSON, 2011, p. 5).

Parafraseando algo que escribí (HERNÁNDEZ, 2011) a propósito de las relaciones pedagógicas, los afectos aparecen, se producen, acontecen, cuando tiene lugar una experiencia de encuentro de subjetividades (cómo nos conocemos y narramos) y saberes (cómo nos vinculamos con lo que conocemos). Este encuentro no se puede predecir ni utilizar como atajo para aprender de ciertos dispositivos culturales, este encuentro emerge. Algo similar es lo que ocurre cuando se asume la transformación que tiene lugar en el transcurso de la investigación, del encuentro entre sujetos, si prestamos atención, 
a que se ponen en juego un yo en proceso de devenir que va configurando historias selectivas, y un yo que confronta el poder con/en una narración del presente.

Todo lo cual me lleva a rescatar la noción de compromiso, en la docencia, en la investigación, en la vida social, entendiéndolo, como señala Marina Garcés (2013, p. 11), como "dejarse afectar, dejarse tocar, dejarse interpelar, saberse requerido, verse implicado: entrar en espacios de vida que no podemos aspirar a controlar del todo, implicarnos en situaciones que nos exceden y que nos exigen inventar respuestas que tal vez no teníamos y que seguro que no nos dejarán igual".

Finalmente, quisiera llamar la atención en algo que he señalado más arriba. Que en este artículo lo que comienza como un proyecto de investigación sobre cómo aprenden los docentes de secundaria, se desplaza a la experiencia de construcción de saber compartido de un grupo personas relacionadas con la Escuela (inspectora, orientadora, directora, docentes de educación infantil, primaria, secundaria y universidad) que deciden ponerse en juego en una experiencia de compartir. Este movimiento es, sobre todo, un movimiento de afectos, en la medida en que promueve transformaciones sobre el pensar(se) en el aprender y en las intra-acciones que se generan a partir de la creación de cartografías, además de promover un desplazamiento de la investigación a la formación desde la consideración de ambas como encuentro de sujetos.

\section{Referencias}

ABAKERLI BAPTISTA, M. B. Relaciones entre la Cultura Visual y la Perspectiva Educativa de los Proyectos de Trabajo en un trayecto de formación. Barcelona: Universidad de Barcelona. Tesis doctoral no publicada, 2014.

ATKINSON, D. Art, equality and learning: Pedagogies against the state. Rotterdam: Sense, 2011-

ATKINSON, D.Contemporary Art in Education: The New, Emancipation and Truth. The International Journal of Art \& Design Education, 31(1), 5-18, 2012. doi: $10.1111 / j .1476-8070.2012 .01724 . x$ 
Fernando Hernández-Hernández

BAKKO, M.; MERZ, S. Toward an Affective Turn in Social Science Research? Theorising Affect, Rethinking Methods and (Re)Envisioning the Social. Graduate Journal of Social Science, v.11, n.1, p. 7-14, Feb. 2015.

BIBLIOTECA NACIONAL DE ESPAÑA. Cartografías de lo desconocido. Madrid: Biblioteca Nacional de España, 2017.

BRAIDOTTI, R. Transpositions. On Nomadic Ethics. Cambridge: Polity Press. 2006.

BRAIDOTTI, R. Writing as a nomadic subject. Comparative Critical Studies, v. 11, n. 2-3, p. 163-184, 2014.

CAIXAFORUM. Contemporary Cartographies. Drawing the though. Barcelona: Fundació La Caixa, 2012.

CAMPS, V. El Gobierno de las Emociones. Barcelona: Herder, 2011.

COLL, C. La educación formal en la nueva ecología del aprendizaje: Tendencias, retos y agendas de investigación. In: RODRÍGUEZ-ILLERA, J.L. (comp). Aprendizaje y educación en la sociedad digital. Barcelona: Universitat de Barcelona, 2013. p. 156170.

CVETKOVICH, A. Depression is Ordinary: Public Feelings and Saidiya Hartman's Lose Your Mother'. Feminist Theory, v.13, n. 2, p. 131-146, 2012.

DELEUZE, G.; GUATTARI, F. A thousand plateaus: Capitalism and schizophrenia. London: Continuum, 1980/2004.

. ¿Qué es la filosofía? Barcelona: Anagrama, 1991/1993.

ERSTAD, O.; KUMPULAINEN, K.; MÄKITALO, A.; C SCHRØDER, K. C.; PRUULM, P.; JÓHANNSDÓTTIR, T. Learning across Contexts in the Knowledge Society. Rotterdam: Sense, 2016.

ERSTAD, O.; SEFTON-GREEN, J. (ed.). Identity, Community, and Learning Lives in Digital Age. Cambridge: Cambridge University Press, 2013.

GARCÉS, M. Un mundo en común. Barcelona: Ediciones Bellaterra, 2013. 
GREENBLAT, S. Resonance and Wonder. In: KARP, I.; LEVINE, S.D. (Eds.). Exhibiting Cultures. Washington \& London: Smithsonian Institute Press, 1992. p. 42-56.

GUATTARI, F. Cartografías Esquizoanalíticas. Buenos Aires: Manantial, 2000.

HERNÁNDEZ, F. (coord.). iY luego dicen que la escuela pública no funciona! Investigar con los jóvenes sobre cómo transitan y aprender dentro y fuera de los centros de secundaria. Barcelona: Octaedro, 2017.

HERNÁNDEZ, F. (coord.). ¿Qué nos cuentan los jóvenes? Narraciones biográficas sobre las relaciones de los jóvenes con el saber en la escuela secundaria. Barcelona: Universidad de Barcelona, 2011.

HERNANDEZ, F. (coord.). Aprender a ser en la escuela primaria. Barcelona: Octaedro, 2010.

HERNANDEZ-HERNANDEZ， F.; SANCHO-GIL， J. M.; DOMINGO-COSCOLLOLA， M. Cartographies as spaces of inquiry to explore of teachers' nomadic learning trajectories. Digital Educational Review, n. 33, p. 105-119, 2018.

HICKEY-MOODY, A. A Femifesta for Posthuman Art Education: Visions and Becomings. In: TAYLOR, C.; HUGHES, C. (eds.). Posthuman Research Practices in Education. UK: Palgrave Macmillan, 2016. p. 258-266.

JACKSON, A. Y.; MAZZEI, L. A. Thinking with theory in qualitative research: Viewing data across multiple perspectives. New York: Routledge, 2012.

KAUFMANN, T. Arte y conocimiento: rudimentos para una perspectiva descolonial. Instituto Europeo para políticas culturales progresistas, 2011. Bajado de: $<$ http://eipcp.net/transversal/0311/kaufmann/es/\# ftn2>.

LATHER, P. Methodology-21: what do we do in the afterward? International Journal of Qualitative Studies in Education, v. 26, n. 6, p. 634-645, 2013.

LATHER, P.; ST. PIERRE, E. A. Post-qualitative research. International Journal of Qualitative Studies in Education, v. 26, n. 6, p. 629-633, 2013. 
MCKINNON, I. Expanding Cartographic Practices in the Social Sciences. In: MARGOLIS, E.; PAUWELS, L.L. (Eds.). The SAGE Handbook of Visual Research Methods. London: SAGE, 2011. p. 452-473.

ONSÉS, J. Subjective Cartographies: Mapping the network of neighbourhood relationships in the neighbourhood of Poble Sec. Conference Mapping Culture. Communities, Sites and Stories. Universidade de Coimbra, Coimbra (Portugal). May 28-30, 2014.

PAULSTON, R. G.; LIEBMAN, M. An Invitation to Postmodern Social Cartography. Comparative Education Review, v. 38, n. 2, p. 215-232, 1994.

RICHARDSON, L.; ST. PIERRE, E. A. Writing: A method of inquiry. In: DENZIN, N.; LINCOLN, Y. S. (Eds.). The SAGE handbook of qualitative research (3rd ed.). Thousand Oaks, CA: Sage, 2005. p. 959-978.

RUITENBERG, C. Here be dragons: Exploring Cartography in Educational Theory and Research. Complicity: An International Journal of Complexity and Education, v.4, p. 7$24,2007$.

SPINOZA, B. Ética demostrada según el orden geométrico. México: Fondo de Cultura, $1677 / 1980$.

ST. PIERRE, E. A. Post Qualitative Research. The Critique and Coming After. In: DENZIN, N.; LINCOLN, Y.S. (Eds.). The SAGE Handbook of Qualitative Research. (3rd ed.). Thousand Oaks, CA: SAGE, 2011. p. 611-625.

SUlLIVAN, G.; GU, M. The possibilities of Research-The promise of Practice. Art Education, v.70, n. 2, p. 49-57, 2017.

ULMER, J. B.; KORO-LJUNGBERB, M. Writing Visually Through (Methodological) Events and Cartography. Qualitative Inquiry, v.2, n. 2, p. 138-152, 2015.

WARF, B.; ARIAS, S. The Spatial Turn: Interdisciplinarity Perspectives. London and New York: Routledge, 2008.

\footnotetext{
' Hernández-Hernández es profesor de Visualidades Contemporáneas, Psicología del Arte e Investigación basada en el Arte en la Unidad de Pedagogías Culturales de la Facultad de Bellas Artes de la Universidad de Barcelona. Es coordinador del Programa de
} 
Postgrado en Artes y Educación. Es co-cordinador del grupo de investigación ESBRINA Subjetividades, visualidades y entornos educativos contemporáneos (2017 SGR 1248) http://esbrina.eu ; y miembro de REUNI+D - Red Universitaria de Investigación e Innovación Educativa. Cambio social y desafíos para la educación en la era digital (EDU2015-68718-REDT) http://reunid.eu

Como citar esse artigo:

HERNÁNDEZ, Fernando Hernández. Encuentros que afectan y generan saber pedagógico entre docentes a través de cartografías visuales. Revista Digital do LAV, Santa Maria: UFSM, v. 11, n. 2, p. 103-120, mai./ago. 2018. 